\title{
hnRNP A1 and hnRNP H can collaborate to modulate 5 ' splice site selection
}

\author{
JEAN-FRANÇOIS FISETTE, ${ }^{1}$ JOHANNE TOUTANT, ${ }^{1}$ SAMUEL DUGRÉ-BRISSON, ${ }^{2}$ LUC DESGROSEILLERS, ${ }^{2}$ \\ and BENOIT CHABOT ${ }^{1}$ \\ ${ }^{1}$ Département de Microbiologie et d'Infectiologie, Faculté de Médecine et des Sciences de la Santé, Université de Sherbrooke, Sherbrooke, \\ Québec H3C 3J7, Canada \\ ${ }^{2}$ Département de Biochimie, Université de Montréal, Montréal, Québec H3C 3J7, Canada
}

\begin{abstract}
The mammalian proteins hnRNP A1 and hnRNP H control many splicing decisions in viral and cellular primary transcripts. To explain some of these activities, we have proposed that self-interactions between bound proteins create an RNA loop that represses internal splice sites while simultaneously activating the external sites that are brought in closer proximity. Here we show that a variety of hnRNP H binding sites can affect $5^{\prime}$ splice site selection. The addition of two sets of hnRNP $H$ sites in a model pre-mRNA modulates 5' splice site selection cooperatively, consistent with the looping model. Notably, binding sites for hnRNP A1 and $\mathrm{H}$ on the same pre-mRNA can similarly collaborate to modulate $5^{\prime}$ splice site selection. The C-terminal portion of hnRNP $\mathrm{H}$ that contains the glycine-rich domains (GRD) is essential for splicing activity, and it can be functionally replaced by the GRD of hnRNP A1. Finally, we used the bioluminescence resonance energy transfer (BRET) technology to document the existence of homotypic and heterotypic interactions between hnRNP H and hnRNP A1 in live cells. Overall, our study suggests that interactions between different hnRNP proteins bound to distinct locations on a pre-mRNA can change its conformation to affect splicing decisions.
\end{abstract}

Keywords: hnRNP A1; hnRNP H; synergy; alternative splicing; looping out

\section{INTRODUCTION}

As many as $95 \%$ of human multiexon genes may be alternatively spliced (Pan et al. 2008; Wang et al. 2008). Alternative splicing therefore represents an important mechanism to increase proteome diversity, hence contributing to the biological complexity of mammals (Graveley 2001). Appropriate selection of alternative splice sites is critical to produce proteins with specific functional attributes. The orchestration of this multitude of splicing decisions necessitates RNA binding proteins of which members of the SR and hnRNP protein families are the best characterized.

SR proteins play a role both in constitutive and alternative splicing (Graveley 2000). This family of proteins frequently enforces exon inclusion by binding to exonic

Reprint requests to: Benoit Chabot, Département de Microbiologie et d'Infectiologie, Faculté de Médecine et des Sciences de la Santé, Université de Sherbrooke, Sherbrooke, Québec H3C 3J7, Canada; e-mail: Benoit. Chabot@USherbrooke.ca; fax: (819) 564-5392.

Article published online ahead of print. Article and publication date are at http://www.rnajournal.org/cgi/doi/10.1261/rna.1890310. splicing enhancers to stimulate the binding of generic splicing factors including U2AF, U2 snRNP, and U1 snRNP (Lavigueur et al. 1993; Kohtz et al. 1994; Wang et al. 1995; Zuo and Maniatis 1996; Graveley et al. 2001). However, SR proteins can also have negative impacts on splicing. This is the case in the hnRNP A1 pre-mRNA where SRp30c can repress the $3^{\prime}$ splice site of exon 8 (Simard and Chabot 2002; Paradis et al. 2007), and in the adenovirus L1 unit where ASF/SF2 can prevent the recruitment of U2 snRNP (Kanopka et al. 1996).

On the other hand, hnRNP proteins have been regarded mostly as splicing repressors. Indeed, hnRNP A1 can interfere with the binding of the SR protein SC35 preventing it to stimulate the inclusion of HIV tat exon 2 (Zahler et al. 2004). Moreover, hnRNP Al can inhibit the inclusion of an alternative exon in human Ras, SMN2, $\beta$-tropomyosin, and $c$-src genes (Guil et al. 2003; Kashima and Manley 2003; Rooke et al. 2003; Expert-Bezancon et al. 2004). Likewise, hnRNP $\mathrm{H}$ can repress exon inclusion in several genes: HIV tat, $\alpha$-tropomyosin, $c$-src, and fibroblast growth factor receptor 2 (Min et al. 1995; Chou et al. 1999; Jacquenet et al. 2001; Crawford and Patton 2006; Mauger 
et al. 2008). However, positive roles for hnRNP proteins have also been observed. Intronic binding sites for hnRNP A1 and hnRNP $\mathrm{H}$ can stimulate in vitro splicing (MartinezContreras et al. 2006). In a recent analysis using RNA interference and a RT-PCR screening platform, we examined the role of the major hnRNP proteins in the splicing of apoptotic genes (Venables et al. 2008). Our study suggests that $\sim 10 \%$ of all alternative splicing events are controlled by hnRNP Al and hnRNP H (Venables et al. 2008). Moreover, the effects obtained by knocking down these and other hnRNP proteins suggest that hnRNP proteins can promote a mixture of exon inclusion/exclusion events, and that the identity of the target genes varies considerably in different cellular contexts.

The enhancing or silencing activity of hnRNP A1 and hnRNP $\mathrm{H}$ in splicing vary depending on the position of their respective binding sites relative to the splicing signals. For example, in HIV-1 tev exon 6D, an hnRNP H binding site located 66 nucleotides from the $5^{\prime}$ splice site promotes U1 snRNP binding (Caputi and Zahler 2002), while in the NF- 1 and TSH $\beta$ genes, binding of hnRNP $\mathrm{H}$ at the $5^{\prime}$ splice site restricts U1 snRNP binding (Buratti et al. 2004). hnRNP A1 binding sites can stimulate splicing between an external pair of splice sites while decreasing the use of an intervening one (Nasim et al. 2002; Martinez-Contreras et al. 2006). To explain the role of hnRNP A1 and $H$ binding sites in alternative splicing we have proposed the looping out model, which posits that bound hnRNP proteins self-interact to loop out and repress internal splice sites, while simultaneously juxtaposing and stimulating the use of an external pair of splice sites (Chabot et al. 1997; Blanchette and Chabot 1999; Martinez-Contreras et al. 2006). Consistent with this model, inverted repeats can mimic the impact of A1 binding sites (Nasim et al. 2002). The enrichment of putative binding sites for hnRNP A1 and hnRNP $\mathrm{H}$ proteins (GGG motifs) near both ends of introns is also consistent with a role for this mechanism in generic splicing (Nussinov 1987, 1988, 1989; Engelbrecht et al. 1992; Solovyev et al. 1994; McCullough and Berget 1997; Majewski and Ott 2002; Yeo et al. 2004).

hnRNP Al and hnRNP $\mathrm{H}$ are structurally related proteins. hnRNP A1 contains two N-terminal RNA recognition motifs (RRM1 and RRM2), while the equivalent region in hnRNP $\mathrm{H}$ hosts two motifs that display small similarity with the classical RRM but can bind RNA and were denoted quasi-RRMs (qRRM1 and qRRM2) (Honore et al. 1995). The C-terminal half of hnRNP A1 contains a glycine-rich domain (GRD) that is required for selfinteraction (Cartegni et al. 1996) and is essential for the activity of A1 in splicing (Mayeda et al. 1994). hnRNP H contains two GRDs interrupted by a qRRM (qRRM3). The function of the individual domains of hnRNP $\mathrm{H}$ has not yet been investigated. Given their structural similarity and their equivalent impact on splicing, it was of interest to determine if hnRNP Al and hnRNP $\mathrm{H}$ could collaborate to enforce a specific splicing decision. Our in vitro analysis indicates that they do and their very close proximity in live cells suggests a direct interaction between hnRNP Al and hnRNP H, consistent with the looping out model of action.

\section{RESULTS}

\section{hnRNP H binding sites modulate $5^{\prime}$ splice site selection}

Using a model pre-mRNA that contains two $5^{\prime}$ splice sites competing for a single $3^{\prime}$ splice site, we have shown previously that inserting a hnRNP $\mathrm{H}$ binding site downstream from each of two competing 5 ' splice sites stimulates splicing to the upstream one in vitro (MartinezContreras et al. 2006). The hnRNP H binding site used in our previous study was from the cystathionine $\beta$-synthase gene where it regulates $3^{\prime}$ splice site selection (Romano et al. 2002). This site, here called HBS1, is bound by hnRNP $\mathrm{H}$ but not by hnRNP A1 (Martinez-Contreras et al. 2006). There are two $G$ quadruplets present in HBS1 (Fig. 1A, bottom panel). Both $\mathrm{G}$ motifs contribute to the activity of this element since mutating either one or both $G$ quadruplets reduced distal $5^{\prime}$ splice site utilization (Fig. 1B, cf. lanes 4,5,6 and lane 3 ). Thus, and consistent with a role for hnRNP H, maximal activity of HBS1 requires the integrity of $\mathrm{G}$ quadruplets.

As seen for hnRNP A1 binding sites (Blanchette and Chabot 1999), the strongest impact on $5^{\prime}$ splice site selection is observed when HBS1 is placed downstream from both $5^{\prime}$ splice sites (Martinez-Contreras et al. 2006). We have proposed that this strong shift in $5^{\prime}$ splice site selection occurs because bound hnRNP H proteins can selfinteract. This interaction would loop out the internal $5^{\prime}$ splice site and bring in closer proximity the distal pair of splice sites. In its simplest form, the looping out model therefore predicts that the activity of HBS1 should be observed only when HBS1 sites are occupying the two positions on the pre-mRNA. Consistent with the model, placing $\mathrm{HBS} 1$ at both positions stimulated distal $5^{\prime}$ splice site usage (Fig. 1C, lane 7), and having one HBS1 at either the upstream or the downstream position had very little effect (Fig. 1C, cf. lanes 4,5 and lane 1). We also tested a shorter version of HBS1 that contains only one $G$ quadruplet (HBS4). hnRNP $\mathrm{H}$ binds more efficiently to HBS1 than to HBS4 (Supplemenal Fig. 1). Although, placing HBS4 at either position did not improve distal 5' splice site utilization (Fig. 1C, lanes 2,3), HBS4 could cooperate with HBS1 to shift 5 ' splice site selection toward the distal site (Fig. 1C, lanes 6,9).

Although the above experiment illustrates the synergy provided by two HBS located at different positions on a pre-mRNA, we find that individual HBS can often display some activity in different preparations of HeLa extracts (see, for example, Fig. 1E, lanes 1-4). As proposed previously 
A
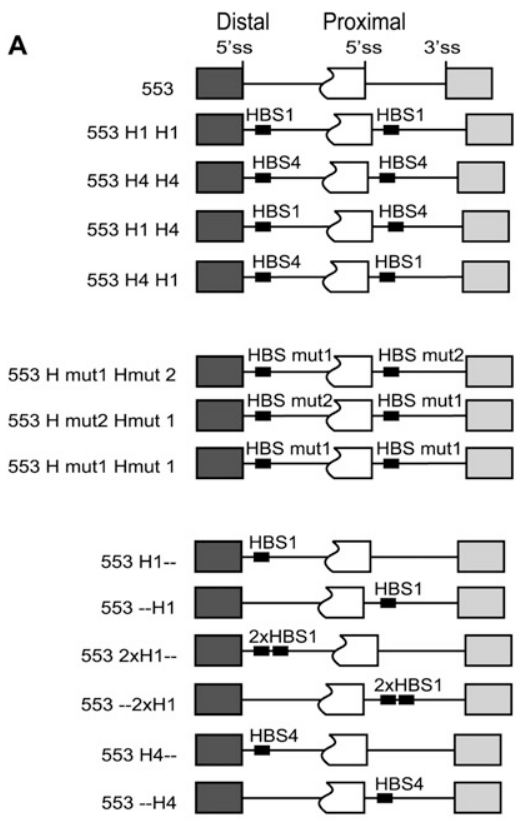

$\begin{array}{ll}\text { HBS1 } & \text { ccauggaucacugggguggaucauccagguggggcuuuu } \\ \text { HBS4 } & \text { ccauggaucacugggguggaucau } \\ \text { HBS mut1 } & \text { ccauggaucacugugguggaucauccagguguggcuuuu } \\ \text { HBS mut2 } & \text { ccauggaucacugugguggaucauccagguggggcuuuu }\end{array}$

B

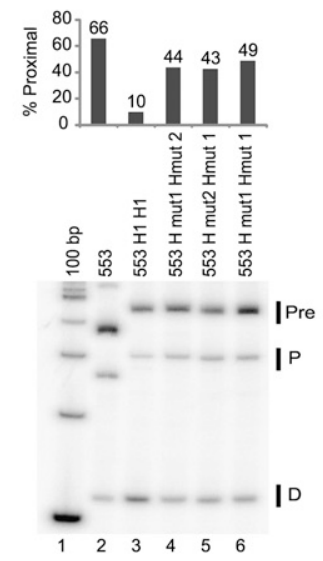

C
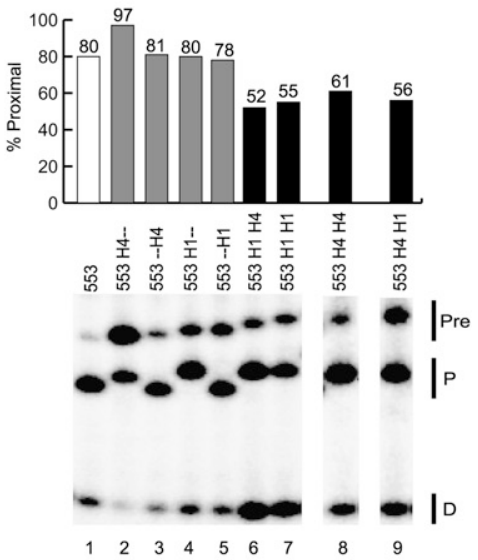

D

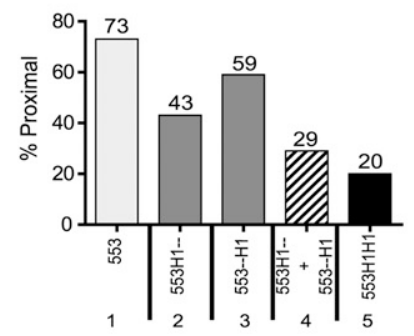

E

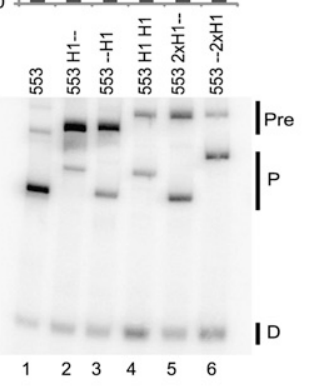

FIGURE 1. Impact of G-tracts on distal $5^{\prime}$ splice site utilization. (A) Schematic representation of pre-mRNAs containing HBS4, HBS1, or mutated HBS1 (HBSmut1 and HBSmut2). (B-E) Pre-mRNAs were incubated in HeLa nuclear extract at $30^{\circ} \mathrm{C}$ for $2 \mathrm{~h}$ and splicing products were amplified by RT-PCR. Amplicons were separated on gels and splicing products corresponding to distal $(D)$ or proximal $(P) 5^{\prime}$ splice site usage or to pre-mRNAs (Pre) are indicated in panels $B, C$, and $E$. The 100-bp caption in panel $B$ indicates molecular weight markers. The percentage of proximal products relative to the sum of the proximal and distal products was calculated. Each splicing reaction performed in panel $B$ was done in triplicate. The $P$-value for the difference between the wild-type $553 \mathrm{H} 1 \mathrm{H} 1$ and each mutant was smaller than 0.05 . In panel $D$, the splicing assays were done in the presence of $10 \mathrm{pmol}$ of DNA oligo HUMMK containing hnRNP H binding sites. The sum of the impact of HBS1 at the individual position (in percentage of shift) has been calculated and is reported in the hatched histogram.

for hnRNP A1 binding sites (Chabot et al. 2003), the activity of a single HBS may be due to weaker hnRNP $\mathrm{H}$ binding sites on the pre-mRNA that provide partners for $\mathrm{H}$ bound to the high-affinity site. Higher concentrations of available hnRNP proteins may cause this activity, and these concentrations may vary in different extracts. When a single
HBS displays activity, it is often more difficult to show the synergy between two high-affinity sites since the sum of the shifts obtained with individual sites is often similar to the shift obtained with the combination. To reduce the contribution of weaker sites, we carried out splicing reactions in the presence of small quantities of a DNA oligo 
(HUMMK) containing hnRNP $\mathrm{H}$ binding sites to reduce the concentration of available hnRNP H in the extract. This site was taken from the polyadenylation signal in the HUMMK gene (Arhin et al. 2002). The HUMMK DNA oligo (CCATGGTTTGGGAGTGGGAAGGTGGGGAG) is bound by recombinant hnRNP H but not A1 (Supplemental Fig. 2). The rationale for this experiment is that if having two binding sites greatly increases the binding affinity, then a competitor would reduce the effect of the single binding site without altering the impact of the double sites. We performed in vitro splicing assays on pre-mRNAs carrying HBS1 in the presence of HUMMK, and the percentages of proximal products were calculated for each reaction. As seen in Figure 1D, the presence of two HBS1 on the same pre-mRNA was stronger than the sum of the impact of individual HBS1 (difference of 53\% between 553 and $553 \mathrm{H} 1 \mathrm{H} 1$ compared with $44 \%$ for the sum of the individual differences [i.e., $30 \%$ for $553 \mathrm{H} 1-$ and $14 \%$ for 553-H1] relative to the control 553). These results again support the view that the two HBS1 act cooperatively, consistent with the looping model.

Another result that rules out simple additive effects is that insertion of two consecutive HBS1 at either position (as depicted in Fig. 1A) produces a shift that is less important (Fig. 1E, lanes 5,6, differences of $18 \%, 27 \%$ with 553 , respectively) than the shift obtained when one HBS1 occupies each site (Fig. 1E, lane 4, difference of 55\%).

To confirm the results obtained with HBS1 and HBS4, we tested the activity of a different binding site for hnRNP H. HBS3 contains a single GGGGG sequence (Fig. 2A) and it is part of a polyadenylation signal in the RATCRP2A gene (Arhin et al. 2002). Since GGG represents the core sequence found in many binding sites for hnRNP H (Caputi and Zahler 2001), but is also part of the high-affinity binding site for hnRNP A1 (Burd and Dreyfuss 1994), we first assessed if HBS3 was bound specifically by hnRNP $\mathrm{H}$. Using a filter binding assay, we show that HBS3 is bound more efficiently by recombinant hnRNP $\mathrm{H}$ than by hnRNP A1 (Fig. 2B). In contrast, the hnRNP A1 binding site CE1a, which exists in the hnRNP Al premRNA (Chabot et al. 1997; Hutchison et al. 2002), is bound by hnRNP Al but not $\mathrm{H}$ (Fig. 2B). Next, we tested if HBS3 could modulate alternative $5^{\prime}$ splice site utilization in the same manner as HBS1. When individual sites were tested, a shift to the distal $5^{\prime}$ splice site was observed in the graph. only when HBS3 was at the upstream position (Fig. 2C, lanes 1-3). However, although HBS3 at the downstream position did not stimulate distal $5^{\prime}$ splice site utilization, it could synergize with a HBS3 at the other position to abrogate splicing to the proximal $5^{\prime}$ splice site almost completely (Fig. 2C, lane 4). Here again, the fact that two HBS3 are more active than the sum of individual sites (51\% shift versus $18 \%$, respectively) supports a cooperative model of action. In our view, the looping out model represents the simplest model that can explain how HBS positioned downstream from either or both of the competing $5^{\prime}$ splice sites always stimulates splicing to the upstream site.

\section{Combining hnRNP A1 and hnRNP H binding sites}

Our in vitro splicing assays show that having ABS or HBS at two separate positions on a pre-mRNA can have a strong effect on 5' splice site selection. The hnRNP Al and hnRNP $\mathrm{H}$ proteins have GRD, and this region in $\mathrm{A} 1$ is essential for self-interaction. If GRDs can promote interactions between different proteins, then $\mathrm{A} 1$ and $\mathrm{H}$ may be able to interact to loop out portions of a pre-mRNA. To test whether HBS and ABS can cooperate to stimulate distal $5^{\prime}$ splice site

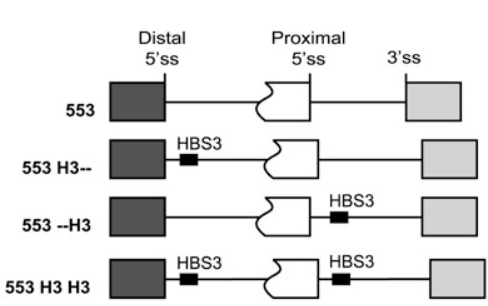

B

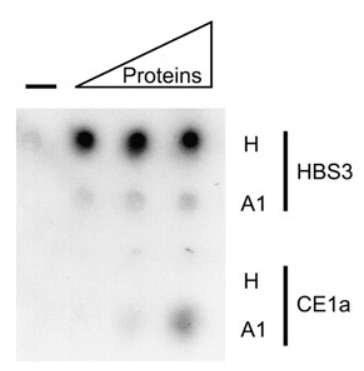

C

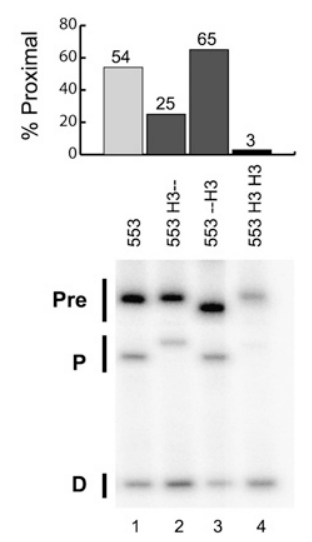

FIGURE 2. A different binding site for hnRNP H can modulate splice site selection. $(A)$ Sequence of HBS3 and the hnRNP A1 binding site CE1a (left). Structure of the pre-mRNAs containing hnRNP $\mathrm{H}$ binding sites (right). (B) hnRNP $\mathrm{H}$ binds more efficiently to HBS3 compared with hnRNP A1. Filter binding assay using ${ }^{32} \mathrm{P}$-labeled HBS3 or CE1a RNAs. Histagged hnRNP $\mathrm{H}$ or $\mathrm{A} 1$ proteins were used at $0,0.5,1$, and $2 \mu \mathrm{M} .(C)$ In vitro splicing assays and RT-PCR amplification. The distal $(D)$, and proximal $(P)$ products as well as the premRNAs (Pre) are indicated. For each reaction, the percentage of proximal product is indicated 
usage, we constructed model pre-mRNAs carrying ABS combined with HBS1 or HBS4 (Fig. 3A). Figure 3B confirms the activity of individual ABS (Fig. 3B, lanes 7,10) and HBS1 (Fig. 3B, lanes 5,6). As observed above, we could not detect a shift with individual HBS4 (Fig. 3B, lanes 3,4). Combining one ABS with HBS1 or HBS4 in different configurations ( $\mathrm{H} 1 \mathrm{~A} 1, \mathrm{~A} 1 \mathrm{H} 1$, and $\mathrm{A} 1 \mathrm{H} 4)$ stimulated distal $5^{\prime}$ splice site usage (Fig. 3B, lanes 9,11,12, 51\%, 70\%, 57\% shifts, respectively). The amplitudes of these shifts were superior to the sum of the shifts imposed by individual ABS or $\operatorname{HBS}(46 \%, 41 \%$, and $34 \%$ shifts, respectively). The probability that such a difference occurred by chance is $<5 \%$ ( $P$-value of 0.045$)$. The amplitude of the shift obtained the H4A1 combination (Fig. 3B, lane 8) suggests that HBS4 at the upstream position provides very little

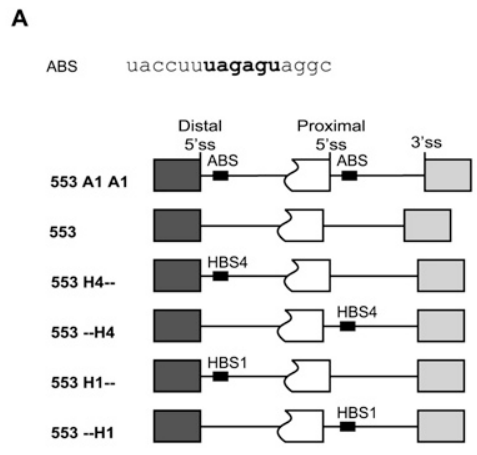

B

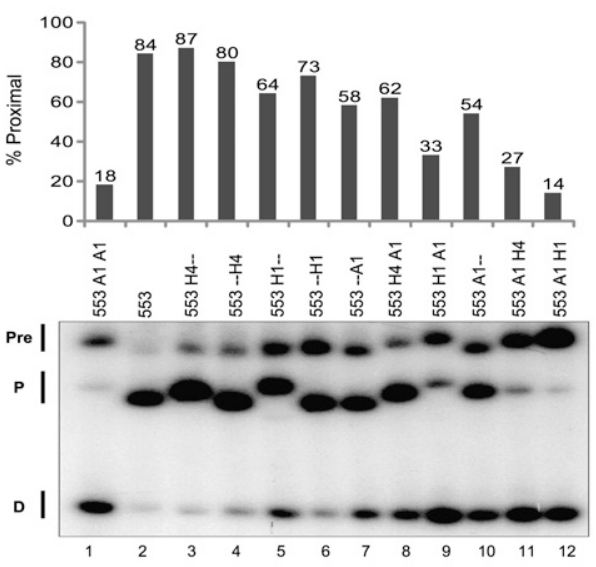

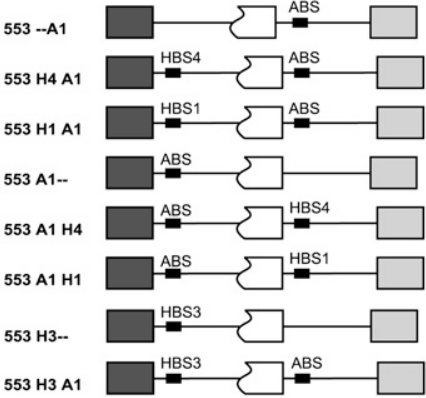

C

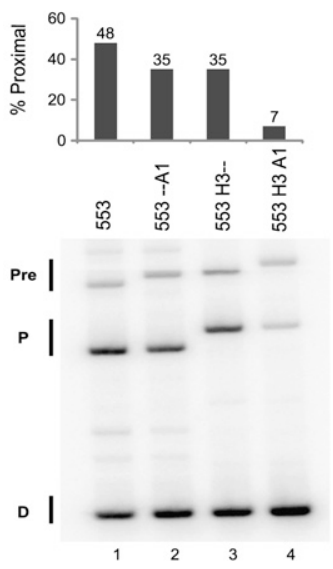

FIGURE 3. HBS and ABS function together to modulate distal $5^{\prime}$ splice site usage. $(A)$ Sequence of ABS and structure of the 553 splicing units containing HBS1, HBS3, HBS4, and ABS. (B) The pre-mRNAs were incubated in HeLa nuclear extract. Pre-mRNAs and splicing products were amplified by RT-PCR. For each reaction, the percentage of proximal product is indicated in the graph. $(C)$ In vitro splicing assays in the presence of 2 pmol of DNA oligo HUMMK containing hnRNP $\mathrm{H}$ binding sites (CCATGGTTTGGGAGTGGGAAGGTGGG GAG) and 2 pmol of DNA oligo TS10 carrying hnRNP A1 binding sites [(TTAGGG) ${ }_{10}$ ]. The sum of the activity of one binding site for hnRNP H or hnRNP Al at both positions was less than the activity of a combination of hnRNP $\mathrm{H}$ and hnRNP A1 binding sites. modulatory activity. These results indicate that the two positions are not equivalent, possibly reflecting contextual differences (e.g., positions of the weaker binding sites and/ or local secondary structures differentially affecting the binding of hnRNP proteins). Overall, however, our results clearly suggest that A1 can cooperatively interact with $\mathrm{H}$ to modulate splicing decisions.

We also tested the impact of combining HBS3 with ABS. In this case, the impact of the $\mathrm{H} 3 \mathrm{~A} 1$ configuration was milar to the sum of the individual activities (data not own). To minimize the impact of weaker binding sites hnRNP $\mathrm{H}$ and $\mathrm{A} 1$, we repeated the assay by includa small amount of the competing oligonucleotides HUMMK and TS10. TS10 contains 10 copies of binding sites for hnRNP A1 ([TTAGGG] $]_{10}$ ) (Dallaire et al. 2000). In these conditions, the impact of the combined sites was superior than the sum of the effect of the individual sites ( $41 \%$ versus $26 \%$ shift, respectively) (Fig. 3C). Thus, different hnRNP $\mathrm{H}$ binding sites can function in synergy with a hnRNP Al binding site to control $5^{\prime}$ splice site selection.

\section{Modularity of the hnRNP A1 and $\mathrm{H}$ domains}

The A1-mediated effects on 5' splice site selection require the GRD of hnRNP A1 (Mayeda et al. 1994; Blanchette and Chabot 1999). In a manner similar to the GRD of the splicing regulator Sxl (Wang et al. 1997), the GRD of A1 may be crucial for mediating cooperativity between multiple A1 proteins bound at distinct locations. Given the equivalent activity of ABS and HBS and the domain similarity between the hnRNP H and $\mathrm{A} 1$ proteins, the GRDs of $\mathrm{H}$ and $\mathrm{A} 1$ may be interchangeable. We tested this hypothesis by constructing chimeric proteins (Fig. 4A,B). First, we assessed the binding capacity of these proteins using a gel shift assay. The GRDqRRM3-GRD portion of hnRNP $\mathrm{H}$ was not essential for RNA binding (Hs) (Fig. 4C, Hs, lanes 8,9). Moreover, linking the qRRM1-qRRM2 portion of hnRNP $\mathrm{H}$ with the GRD of A1 (HA1) preserved binding (Fig. 4C, lanes 12,13). In contrast, the hnRNP F protein, which is $78 \%$ identical to hnRNP $\mathrm{H}$ (Caputi and Zahler 2001), did not bind to HBS1 (Fig. 4C, lanes 4,5). Removing the GRD-qRRM3-GRD portion of hnRNP 
A
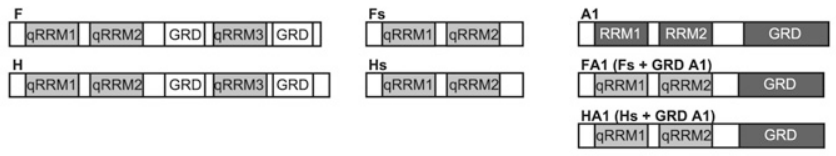

B

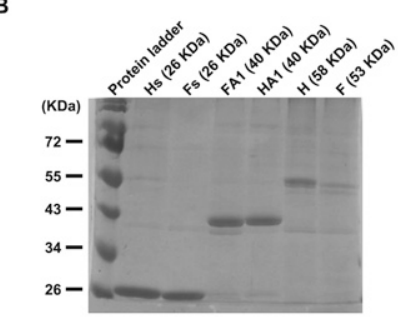

D

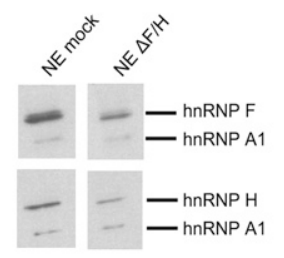

c

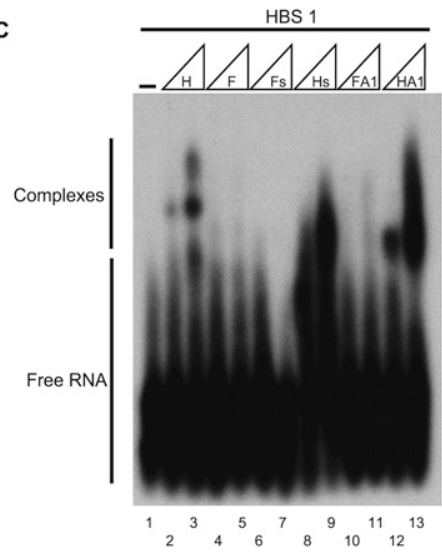

E

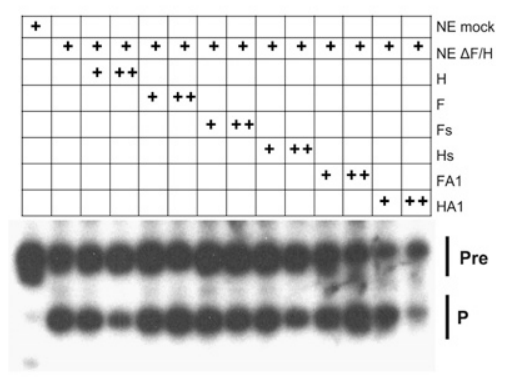

$\begin{array}{llllllllllllll}1 & 2 & 3 & 4 & 5 & 6 & 7 & 8 & 9 & 10 & 11 & 12 & 13 & 14 \\ 17 & 52 & 43 & 34 & 59 & 53 & 59 & 64 & 57 & 51 & 56 & 48 & 28 & 13\end{array}$

FIGURE 4. Swapping domains in the hnRNP A1 and H proteins. (A) Representation of wildtype and chimeric proteins. $(B)$ SDS-PAGE analysis of coomassie-blue stained purified proteins. (C) Binding of recombinant hnRNP H, F, Fs, Hs, FAl, and HAl to ${ }^{32}$ P-labeled HBS1 RNA was monitored by gel shift assay; $0,0.5$, and $1.22 \mu \mathrm{M}$ of recombinant proteins were tested. (D) Western blot analysis after depletion of hnRNP F and hnRNP H. (E) Pre-mRNAs were incubated in a nuclear extract prepared from HeLa cells knocked down for hnRNP F/H expression and containing recombinant proteins $(0.15 \mu \mathrm{M}$ and $0.3 \mu \mathrm{M})$. Distal products (D), proximal products $(\mathrm{P})$, and pre-mRNAs (Pre) are indicated.

F or substituting it for the GRD of A1 did not promote RNA binding (Fig. 4C, lanes 6,7,10,11).

We then tested the splicing activity of the chimeric proteins. The fact that the $553 \mathrm{H} 1 \mathrm{H} 1$ pre-mRNA is spliced mostly to the distal $5^{\prime}$ splice site (Fig. 4E, lane 1) in standard extracts leaves very little latitude for monitoring the impact of exogenous hnRNP proteins. To circumvent this problem, we treated HeLa cells with siRNAs against hnRNP F/H before making nuclear extracts (Fig. 4D). In such conditions, the percentage of proximal products derived from the $553 \mathrm{H} 1 \mathrm{H} 1$ pre-mRNA increases substantially and is strongly reduced by the addition of recombinant his-tagged hnRNP $\mathrm{H}$ protein (Fig. 4E, lanes

2-4). Supplementation with the truncated Hs protein that lacks the GRDqRRM3-GRD region had no effect (Fig. 4E, lanes 9,10), as hnRNP F (Fig. 4E, lanes 5,6), its truncated derivative (Fig. $4 \mathrm{E}$, lanes 7,8$)$, or the chimeric FA1 protein (Fig. 4E, lanes 11,12). Remarkably, the HA1 protein restored distal 5' splice site selection, even to a level that was superior to that of hnRNP H (Fig. 4E, cf. lanes 13,14 and lanes 3,4), possibly reflecting its higher binding affinity for HBS1 or an improved capacity at forming protein-protein interactions. Thus, the GRD-qRRM3GRD portion of hnRNP $\mathrm{H}$, although not required for RNA binding to a G-rich RNA oligo is essential for splicing activity. Moreover, the GRD domain of hnRNP A1 can functionally substitute for the GRD-qRRM3-GRD domain of hnRNP $\mathrm{H}$ in a $5^{\prime}$ splice site selection assay in vitro.

\section{Interaction between hnRNP A1 and hnRNP $H$ in live cells}

Our in vitro splicing results suggest that hnRNP $\mathrm{Al}$ and $\mathrm{H}$ proteins can interact to modulate splice site selection. Distribution of G-triplets in the genome shows a bias toward enrichment in introns (Nussinov 1987, 1988, 1989; Engelbrecht et al. 1992; Solovyev et al. 1994; McCullough and Berget 1997; Majewski and Ott 2002; Yeo et al. 2004; Han et al. 2005). This large quantity of GGG triplets indicates a multitude of putative binding sites for $\mathrm{A} 1$ and $\mathrm{H}$ and, hence, possibilities of interactions between these proteins must be enormous. A recent study using TAP-tagged A1 and $\mathrm{H}$ revealed that at least a portion of these proteins could exist in the same complexes (Jeronimo et al. 2007), but their spatial relationship and proximity were not addressed. To test whether $\mathrm{A} 1$ and $\mathrm{H}$ can interact with one another in a cell, we used the bioluminescence resonance energy transfer (BRET) technology. BRET is based on energy transfer from a donor (Renilla luciferase or Rluc) to an acceptor molecule (YFP) and occurs when the donor/acceptor pair is within $50-100 \AA$ (Xu et al. 1999), which is the typical distance separating the centers of two interacting proteins. Proximities between hnRNP $\mathrm{H}$ and hnRNP A1 proteins were tested in EcR 293 cells. An equivalent quantity of plasmids programmed to express Rluc-tagged proteins was 
cotransfected with an increasing amount of plasmids designed to express YFP-tagged proteins (Fig. 5A). The BRET signal is calculated as the ratio of the YFP excited light over luciferase emission. Because of variations in the expression profile of each plasmid, the BRET signal is plotted relative to total YFP activity/Rluc activity, which essentially normalizes YFP expression relative to the Rluc signal in each transfected sample. We first used the BRET assay to confirm the interaction between hnRNP A1 proteins since Al can self-interact in vitro (Cartegni et al. 1996). A strong BRET signal was obtained when Rluc Al was coexpressed in EcR 293 cells with increasing amounts of YFP A1 (Fig. 5B,C), thus indicating that hnRNP A1 can self-interact in vivo. As expected for a negative control, only a very weak signal was obtained when Rluc and YFP

A

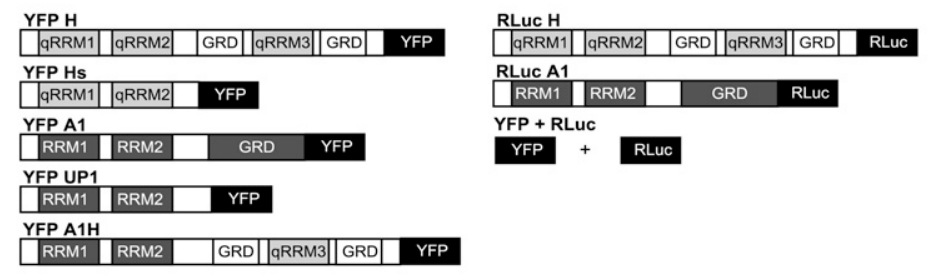

B
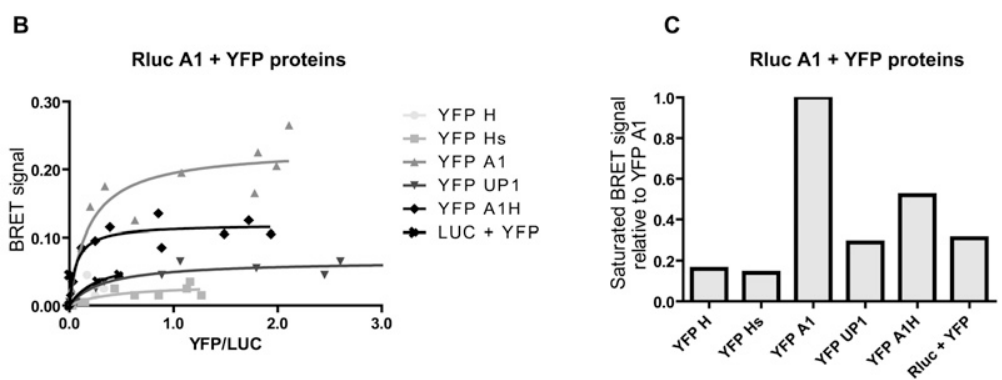

D

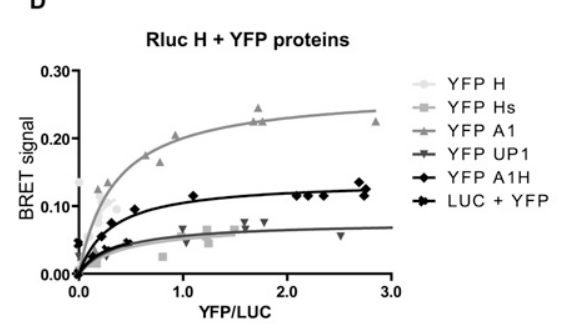

were coexpressed (Fig. 5B,C). Likewise, a similarly low BRET signal was obtained when the GRD of A1 was removed (YFP UP1), indicating that this self-interaction requires the GRD (Fig. 5B,C).

To test whether a similar interaction can occur between hnRNP Al and $\mathrm{H}$, we coexpressed Rluc A1 with YFP H. In this case, the BRET signal was low but this could be due to the low expression of YFP $\mathrm{H}$ (Fig. 5B) and we did not succeed in improving expression. Because a BRET signal often depends on the configuration of the interaction and the design of the interacting proteins (Pfleger et al. 2006), we tested the reciprocal Rluc $\mathrm{H}$ design with YFP A1. Following expression in EcR 293 cells, strong BRET signals were obtained following coexpression (Fig. 5D,E). The result obtained with YFP UP1 indicates that this interaction is dependent on the GRD of A1. Our results therefore indicate that hnRNP $\mathrm{H}$ can interact with hnRNP Al in live cells. Likewise, a strong BRET signal was obtained when Rluc $\mathrm{H}$ was coexpressed with YFP H (Fig. 5D,E). The intensity of the signal dropped considerably when the GRD-qRRM3-GRD domain of $\mathrm{H}$ was removed (YFP Hs; Fig. 5D,E), indicating that the hnRNP H self-interaction is dependent on this region. Overall, the $\mathrm{A} 1-\mathrm{A} 1$ and $\mathrm{A} 1-\mathrm{H}$ interactions were stronger than the $\mathrm{H}-\mathrm{H}$ interaction. This conclusion was confirmed by substituting the GRD portion of A1 for that of $\mathrm{H}$ (YFP A1H); coexpressing Rluc $\mathrm{H}$ and YFP $\mathrm{AlH}$ produced a BRET signal that was in the same order than with YFP H. Overall, our results indicate that homotypic $\mathrm{A} 1-\mathrm{A} 1$ and $\mathrm{H}-\mathrm{H}$ interactions occur in EcR 293 cells and that they require the GRD-containing portions of these proteins. Moreover and importantly, heterotypic interactions between hnRNP $\mathrm{H}$ and hnRNP Al can also occur in live cells.

\section{DISCUSSION}

Our study has investigated the mechanism by which hnRNP H binding sites can modulate $5^{\prime}$ splice site selection. hnRNP $\mathrm{H}$ binding sites containing GGGG or GGGGG in different sequence contexts showed a similar ability at promoting distal $5^{\prime}$ splice site selection using a model pre-mRNA in HeLa extracts. The essential role of $G$ runs was demonstrated since mutating one $G$ in one quadruplet 
was sufficient to abrogate its activity in splice site selection.

The mechanisms by which a hnRNP H protein bound to G-runs control splice site selection can vary. When $G$ runs exist very near splice sites, the binding of hnRNP $\mathrm{H}$ can sterically interfere with splice site recognition (Jacquenet et al. 2001). In contrast, our initial experiments have shown that the presence of hnRNP $\mathrm{H}$ binding sites $30 \mathrm{nt}$ downstream from each of two competing $5^{\prime}$ splice sites shifted the splicing ratio in favor of the upstream splice site (Martinez-Contreras et al. 2006). We proposed that the mechanism of action was similar to the model previously formulated for hnRNP A1 (Blanchette and Chabot 1999; Nasim et al. 2002; Chabot et al. 2003), namely, that bound hnRNP $\mathrm{H}$ proteins would self-interact to loop out and repress the intervening $5^{\prime}$ splice site, while the simultaneous approximation of the distal $5^{\prime}$ and the $3^{\prime}$ splice sites would encourage their commitment. An alternative model based on a recent study on hnRNP A1 (Okunola and Krainer 2009) could be that the binding of hnRNP A1 or $\mathrm{H}$ could spread cooperatively between the high-affinity sites. However, since the splicing shifts that are promoted by highaffinity hnRNP A1 or hnRNP $\mathrm{H}$ sites are not accompanied by a drop in the binding of U1 snRNP to the intervening 5' splice site (Martinez-Contreras et al. 2006), this scenario is unlikely to occur on our pre-mRNAs.

The looping model implies that the strongest stimulation in distal $5^{\prime}$ splice site usage should be observed when appropriately positioned HBS are located downstream from each competing $5^{\prime}$ splice site. Consistent with this view, we showed that consecutive HBS at either the upstream or the downstream position were much less efficient than having one HBS at each position. Moreover, for three different hnRNP $\mathrm{H}$ binding sites, the impact of having binding sites at the two positions was superior to the sum of the impact of sites at individual positions. These results provide support for the looping model. Our model premRNAs have been tested in different preparations of nuclear extracts. In some experiments, a hnRNP $\mathrm{H}$ binding site at only one position can confer some shifting activity. While we cannot rule out that some of this activity may be due to alternative mechanisms (for example, the binding of $\mathrm{H}$ downstream from the proximal $5^{\prime}$ splice site may occlude this site accounting for the shift toward the upstream donor site), our preferred explanation for this behavior is that a protein bound to a single strong site may interact with proteins bound to weaker hnRNP $\mathrm{H}$ sites on the pre-mRNA. Consistent with this view, we have shown that we can reduce the impact of these potentially weaker sites by adding to splicing mixtures small quantities of oligonucleotides that carry binding sites.

Having obtained strong indications that hnRNP $\mathrm{H}$ binding sites can function in a manner similar to those of hnRNP A1, we next asked if $\mathrm{H}$ and A1 binding sites could cooperate to modulate $5^{\prime}$ splice site selection. To answer this question, we produced a battery of pre-mRNA substrates containing combinations of $\mathrm{A} 1$ and $\mathrm{H}$ sites. Relative to individual sites, the combinations nearly always had the strongest impact on $5^{\prime}$ splice site selection. Moreover, in four cases (H1A1, A1H1, A1H4, and $\mathrm{H} 3 \mathrm{~A} 1)$, the effects of the combined sites were superior to the sum of the impact of the individual sites, consistent with the view that hnRNP $\mathrm{H}$ and hnRNP A1 can cooperate to affect $5^{\prime}$ splice selection synergistically.

The capacity of hnRNP Al and hnRNP $\mathrm{H}$ binding sites to cooperate in $5^{\prime}$ splice site selection implies that the A1 and $\mathrm{H}$ proteins can interact with one another. Although at least a fraction of hnRNP A1 and $\mathrm{H}$ proteins exist in similar complexes based on a previous study (Jeronimo et al. 2007), their ability to establish direct interactions has never been documented. We used the BRET technology to show that hnRNP Al and hnRNP $H$ proteins can coexist in very close proximity, suggesting self-interaction and interaction with one another in live mammalian cells. The C-terminal GRD of $\mathrm{Al}$ and $\mathrm{H}$ is important for both homotypic and heterotypic interactions since their removal strongly reduced the BRET signals. Moreover, the GRD of A1 and $\mathrm{H}$ are also essential for splicing activity and replacing the GRD of $\mathrm{H}$ with that of A1 maintained splicing activity.

Recently, we have provided further support for the looping out model by showing that intronic sites for hnRNP A1 or H can stimulate the splicing of longer introns in vitro (Martinez-Contreras et al. 2006). The looping model has since been invoked to explain the activity of hnRNP A1 and other RNA binding proteins in splice site selection on other pre-mRNAs. For example, hnRNP A1 bound to exonic and intronic sites may cooperate to induce exon exclusion in the SMN2 pre-mRNA (Kashima et al. 2007). Also, the tissue-specific regulator Nova may multimerize to form an RNA loop to bring in closer proximity a distant $5^{\prime}$ splice site and a branch site (Ule et al. 2006). Finally, this model may explain the noted cooperativity between exonic UAGG and a 5' splice site-proximal GGGG motif to silence exons (Han et al. 2005).

The capacity of hnRNP Al and hnRNP H to interact and cooperate to modulate splicing decisions has important implications on our view of the mechanisms of splicing control. It means that splicing control may rely heavily on the combinatorial arrangement of hnRNP Al and hnRNP $\mathrm{H}$ binding sites. A capacity for hnRNP Al and hnRNP $\mathrm{H}$ to collaborate may not be too surprising because these proteins display redundant binding specificity and have a similar structure. Our findings also raise the possibility that hnRNP A1 and/or $\mathrm{H}$ entertain interactions with other hnRNP proteins, particularly those carrying GRD (hnRNP F, G, P2, Q, D, U, and R) (Martinez-Contreras et al. 2007). The binding of these proteins at various locations on a premRNA or their association via other RNA binding proteins would expand the ways pre-mRNA conformation could be rearranged to alter splicing decisions. 


\section{MATERIALS AND METHODS}

\section{Plasmid constructs}

Pre-mRNA substrates 553(p45), 553A1-(p42), 553-A1 (p39), and 553A1 A1 (p36) have been described earlier (Nasim et al. 2002). To construct plasmids p553H1- and p553H1 A1, DNA oligonucleotide J1 (5'-CCATGGATCACTGGGGTGGATCATCCAGG TGGGGCTTTT-3') and J2 (5'-AAAAGCCCCACCTGGATGATC CACCCCAGTGATCCATGG-3') were hybridized and inserted into the SmaI sites of p45 and p39, and the EcoRV sites of p45 and $\mathrm{p} 42$ to generate $\mathrm{p} 553-\mathrm{H} 1$ and $\mathrm{p} 553 \mathrm{~A} 1 \mathrm{H} 1$, respectively. The same oligos were also inserted into the SmaI site of plasmids p553-H1 to generate p553H1 H1. Oligonucleotides J3 (5'CCATGGATCACTGGGGTGGATCAT-3') and J4 (5'-ATGATC CACCCCAGTGATCCATGG-3') were hybridized and cloned into the SmaI sites of p45 and p39 or introduced into the EcoRV sites of the p45 and p42 to produce p553H4-, p553H4 A1, p553-H4, and p553A1 $\mathrm{H} 4$, respectively. Plasmid p553-H4 was used as a backbone to introduce the complementary oligos into the SmaI site to generate p553H4 H4. Complementary oligos J5 (5' -CCAT GGTTTGGGGGCAGTAGTTGG-3') and J6 (5'-CCAACTACTGC CCCCAAACCATGG-3') were inserted into the SmaI sites of the p45 and p39 or inserted into the EcoRV site of p45 and p42 to produce p553H3-, p553H3 A1, p553-H3 and p553A1H3, respectively. The same oligos were introduced into the SmaI site of p553-H3 to generate p553H3 H3. Pre-mRNA substrates p553Hmut1 Hmut2, p553Hmut1 Hmut1, and pHmut2 Hmul were constructed using the same strategies presented earlier. Oligos ( $5^{\prime}$-CCATGGATCA CTGGTGTGGATCATCCAGGTGGTGCTTTT- $3^{\prime}$ ) and (5'-CCAT GGATCACTGGTGTGGATCATCCAGGTGGGGCTTTT-3') were hybridized with their complementary molecules and inserted into the SmaI sites of the p45 to generate the intermediate constructs p553Hmut1- and pHmut2-. These inserts were then cloned into EcoRV of p553Hmut1- and pHmut2- to produce p553Hmut1 Hmut2, p553Hmut1 Hmut1, and p553Hmut2 Hmut1.

Mutant cDNAs Hs and Fs were produced by PCR amplification on pVL1392 hnRNP $\mathrm{H}$ and pVL1392 hnRNP F, respectively. Forward primer F/H.1 (5'-CCGCTCCAGAATTCTAGAAA TAATTTTGT- $3^{\prime}$ ) containing XbaI restriction site was used for both constructions. Reverse primer Hstop (5'-TTGGATCCC TACCCAGGTCTGTCA-3') and Fstop (5'-TTGGATCCCTACCC GGGCCG3') containing a BamHI site were used for Hs and Fs, respectively. PCR fragments were cut with BamHI and XbaI and inserted in pVL1392 using the same restriction sites. Hybrid constructions H(qRRM1-2) + A1(GRD) (HA1) were generated by production of two intermediate plasmids. First, a SmaI restriction site was created in plasmid pVL1392 hnRNP H by PCR overlap mutagenesis. A first amplification from pVL1392 hnRNP H by primers F/H.1 and H2smaI (5'-CCCGGGTCTGTCATAAGG-3') and a second amplification from the same template by primers H3SmaI (5'-ACCCGGGGCTGGTAG-3') and H4 (5'-AAGGAT CCCTATGCAATGTTTGATT- $3^{\prime}$ ) were performed. Products were purified and mixed for another PCR reaction using primers F/H.1 and H4. PCR fragment was digested with XbaI and BamHI, cloned in pVL1392 to generate pVL1392 HSmaI. Another intermediate plasmid was created by insertion of an EagI restriction site in $\mathrm{pQE} 80 \mathrm{~L}-\mathrm{A} 1$. PCR was performed from plasmid $\mathrm{pQE} 80 \mathrm{~L}-\mathrm{A} 1$ using forward primer A1.1 (5'-ACCATCACGGATCCCCCATGT CTAAGTTCC-3') and reverse primer A1.2 EagI (5'-ACTGC
GGCCGCGCTGACTGGATGAAGC-3'). Another reaction was performed with primer A1.3 EagI (5'-AGTCAGCGCGGCCGCA GTGGTTCTGG-3') and primer A1.4 (5'-AGCTAATTAAGCTTT GTTTCCTGGCTGTA-3'). The two PCR fragments were purified and mixed for another PCR using primer A1.1 and A1.4. The PCR amplicon was digested with HindIII and BamHI, cloned in PQE80L to generate pQE80L-Al EagI. Fragment HindIII-EagI from this plasmid was removed and treated with Klenow. Plasmid pVL1392 HSmaI was digested with SmaI and BamHI and treated with Klenow. The SmaI-BamHI fragment was replaced with the HindIII-EagI fragment from pQE80L-A1 EagI to generate pVL1392 H-A1.

Plasmid pYFP H expressing YFP in the C-terminal of hnRNP H was made by PCR amplification using pVL1392 hnRNP H and primers BRET H.1 (5' ${ }^{\prime}$ TATCCGGGTACCGAGATGATGTTGGG CAC-3') and BRET YFP H.4 (5'-CGGATAGGATCCGCAATG TTTGATTGAAAATCAC-3'). PCR fragments were purified, cut with KpnI- BamHI and inserted in pYFP-CMV-Topaz using the same restriction sites. The same strategy was applied to create plasmids YFP Hs using primers BRET H.1 and BRET YFP H.5 (5'-CGGATAGGATCCGCCCCAGGTCTG-3'). Amplification on pQE80L-A1 with primers BRET A1.1 (5'-TATCCGGGTACCCC CATGTCTAAGTCCGAG-3') and BRET YFP A1.4 (5'-CGGATA GGATCCAACCTCCTGCCACTG-3') was used to generate plasmids YFP A1, and amplification with primers BRET A1.1 and BRET YFP A1.5 (5'-CGGATAGGATCCCTGCGACCTCTCTGAC-3') to create plasmids YFP UP1. The PCR fragments were inserted in pYFP-CMV-Topaz using the same strategy. We performed a PCR reaction on pVL1392 A1H with primers BRET A1.1 and BRET YFP A1.4, a digestion to produce fragments that were inserted in pYFPCMV-Topaz with the same restriction enzymes to produce YFP A1H. PCR amplification on pVL1392 $\mathrm{H}$ was carried out to create a plasmid that could express Rluc at the C-terminal portion of hnRNP H. Primer BRET H.1 and BRET LUC H.2 (5'-CGGATAG GATCCATGCAATGTTTGATTGAAAATCAC-3') were used. The amplified fragments were than purified, cut with KpnI-BamHI, and inserted in pRluc-N1(h) using the same restriction sites. PCR reactions to generate Rluc A1 used oligos BRET A1.1 and BRET LUC A1.2 (5'-CGGATAGGATCCAGAACCTCCTGCCACTG-3') on $\mathrm{pQE} 80 \mathrm{~L}-\mathrm{A} 1$. The PCR fragments resulting from this reaction were cut and inserted as described previously.

\section{In vitro transcription and splicing assays}

Linearized constructs using ScaI were used as template for in vitro transcription. Pre-mRNA substrates were synthesized and purified as described (Martinez-Contreras et al. 2006). Substrates were incubated in HeLa nuclear extract (Dignam et al. 1983) under standard conditions (Chabot et al. 1997) at $30^{\circ} \mathrm{C}$. Purified RNA were amplified using Ready-To-Go RT-PCR beads (GE Healthcare) as described previously (Nasim et al. 2002). Nuclear extract prepared from HeLa cells knocked down for hnRNP $\mathrm{F} / \mathrm{H}$ expression were produced using siRNA against hnRNP $\mathrm{F} / \mathrm{H}$ (Garneau et al. 2005). Recombinant His-tagged proteins were produced using baculovirus expression system (BD Biosciences) (Garneau et al. 2005).

\section{Gel shift assay}

RNA oligonucleotides (HBS1) were labeled using T4 polynucleotide kinase. RNA molecules were incubated in splicing mix 
(1.4 mM rATP, $9 \mathrm{mM} \mathrm{MgCl}_{2}, 50 \mathrm{mM}$ creatine phosphate, $7 \%$ polyvinylalcohol, $5.5 \mathrm{mM}$ dithiothreitol, and 2 units of RNAguard; GE Healthcare). Recombinant proteins were then added to the mixture and allowed to incubate on ice for $5 \mathrm{~min}$. Heparin at a final concentration of $0.75 \mathrm{mg} / \mathrm{mL}$ and loading dye were added and the final reaction were loaded on a $5 \%$ native acrylamide gel (29:1, acrylamide:bisacrylamide) in Tris-glycine buffer.

\section{Filter binding assay}

RNA oligonucleotides (HBS2, HBS3, and CE1a) were labeled using T4 polynucleotide kinase. After the reaction was completed, quantitated and the RNA purified, cold RNA oligo was added to obtain equivalent-specific activity ( $\mathrm{cpm} / \mathrm{pmole}$ ) for the different labeled RNA oligos. We then incubated these labeled RNAs with the recombinant proteins as previously described for the gel shift assay. Mixtures were then added to a 96-well filtration apparatus (Bio-Rad Bio-Dot) and washed with buffer containing $10 \mathrm{mM}$ HEPES at pH 7.9, $50 \mathrm{mM} \mathrm{KCl,} \mathrm{10 \%} \mathrm{glycerol,} \mathrm{and} 0.1 \mathrm{mM}$ EDTA.

\section{BRET assay}

EcR 293 cells were seeded in 12-well plates at a density of $1.5 \times$ $10^{5}$ cells/well. Twenty-four hours later transfection was accomplished using Lipofectamine 2000 (Invitrogen). Ten nanograms of plasmids Rluc, Rluc H, or Rluc Al were cotransfected with increasing amounts $(50,100,250$, and $500 \mathrm{ng})$ of plasmids YFP, YFP H, YFP Hs, YFP A1, YFP UP1, or YFP A1H. Thirty-six hours post-transfection, cells were collected, washed, and resuspended in $200 \mu \mathrm{L}$ of PBS $1 \times$. Ninety microliters were distributed in a 96well microplate. Expression level of the YFP fused proteins were determined by measurement of fluorescence (YFP) (excitation filter at $485 \mathrm{~nm}$ and detection filter at $550 \mathrm{~nm}$ ). The BRET signal is calculated as the fluorescence/luminescence after addition of $5 \mu \mathrm{M}$ coelenterazine $\mathrm{h}$, which is a substrate for luciferase (emission filter 1 for YFP at $550 \mathrm{~nm}$ and emission filter 2 for Rluc at $470 \mathrm{~nm}$ ). Measurements were realized with the Fusion alpha-FP apparatus (Perkin-Elmer-Canberra, Packard BioScience).

\section{SUPPLEMENTAL MATERIAL}

Supplemental material can be found at http://www.rnajournal.org.

\section{ACKNOWLEDGMENTS}

This study was supported by a grant from the Canadian Institute of Health Research to B.C. B.C. is a Canada Research Chair in Functional Genomics. S.D.-B. was supported by a studentship from the Fonds pour la Recherche en Santé du Québec (FRSQ).

Received August 18, 2009; accepted October 5, 2009.

\section{REFERENCES}

Arhin GK, Boots M, Bagga PS, Milcarek C, Wilusz J. 2002. Downstream sequence elements with different affinities for the hnRNP $\mathrm{H} / \mathrm{H}^{\prime}$ protein influence the processing efficiency of mammalian polyadenylation signals. Nucleic Acids Res 30: 1842-1850.

Blanchette M, Chabot B. 1999. Modulation of exon skipping by highaffinity hnRNP A1-binding sites and by intron elements that repress splice site utilization. EMBO J 18: 1939-1952.
Buratti E, Baralle M, De Conti L, Baralle D, Romano M, Ayala YM, Baralle FE. 2004. hnRNP H binding at the $5^{\prime}$ splice site correlates with the pathological effect of two intronic mutations in the NF-1 and TSH $\beta$ genes. Nucleic Acids Res 32: 4224-4236.

Burd CG, Dreyfuss G. 1994. RNA binding specificity of hnRNP A1: Significance of hnRNP Al high-affinity binding sites in pre-mRNA splicing. EMBO J 13: 1197-1204.

Caputi M, Zahler AM. 2001. Determination of the RNA binding specificity of the heterogeneous nuclear ribonucleoprotein (hnRNP) H/H'/F/2H9 family. J Biol Chem 276: 43850-43859.

Caputi M, Zahler AM. 2002. SR proteins and hnRNP H regulate the splicing of the HIV-1 tev-specific exon 6D. EMBO J 21: 845-855.

Cartegni L, Maconi M, Morandi E, Cobianchi F, Riva S, Biamonti G. 1996. hnRNP A1 selectively interacts through its Gly-rich domain with different RNA-binding proteins. J Mol Biol 259: 337-348.

Chabot B, Blanchette M, Lapierre I, La Branche H. 1997. An intron element modulating $5^{\prime}$ splice site selection in the hnRNP A1 pre-mRNA interacts with hnRNP A1. Mol Cell Biol 17: 17761786.

Chabot B, LeBel C, Hutchison S, Nasim FH, Simard MJ. 2003. Heterogeneous nuclear ribonucleoprotein particle $\mathrm{A} / \mathrm{B}$ proteins and the control of alternative splicing of the mammalian heterogeneous nuclear ribonucleoprotein particle Al pre-mRNA. Prog Mol Subcell Biol 31: 59-88.

Chou MY, Rooke N, Turck CW, Black DL. 1999. hnRNP H is a component of a splicing enhancer complex that activates a c-src alternative exon in neuronal cells. Mol Cell Biol 19: 69-77.

Crawford JB, Patton JG. 2006. Activation of alpha-tropomyosin exon 2 is regulated by the SR protein 9G8 and heterogeneous nuclear ribonucleoproteins H and F. Mol Cell Biol 26: 8791-8802.

Dallaire F, Dupuis S, Fiset S, Chabot B. 2000. Heterogeneous nuclear ribonucleoprotein $\mathrm{A} 1$ and UP1 protect mammalian telomeric repeats and modulate telomere replication in vitro. $\mathrm{J}$ Biol Chem 275: 14509-14516.

Dignam JD, Lebovitz RM, Roeder RG. 1983. Accurate transcription initiation by RNA polymerase II in a soluble extract from isolated mammalian nuclei. Nucleic Acids Res 11: 1475-1489.

Engelbrecht J, Knudsen S, Brunak S. 1992. G+C-rich tract in $5^{\prime}$ end of human introns. J Mol Biol 227: 108-113.

Expert-Bezancon A, Sureau A, Durosay P, Salesse R, Groeneveld H, Lecaer JP, Marie J. 2004. hnRNP A1 and the SR proteins ASF/SF2 and SC35 have antagonistic functions in splicing of $\beta$-tropomyosin exon 6B. J Biol Chem 279: 38249-38259.

Garneau D, Revil T, Fisette JF, Chabot B. 2005. Heterogeneous nuclear ribonucleoprotein $\mathrm{F} / \mathrm{H}$ proteins modulate the alternative splicing of the apoptotic mediator Bcl-x. J Biol Chem 280: 2264122650.

Graveley BR. 2000. Sorting out the complexity of SR protein functions. RNA 6: 1197-1211.

Graveley BR. 2001. Alternative splicing: Increasing diversity in the proteomic world. Trends Genet 17: 100-107.

Graveley BR, Hertel KJ, Maniatis T. 2001. The role of U2AF35 and U2AF65 in enhancer-dependent splicing. RNA 7: 806-818.

Guil S, Gattoni R, Carrascal M, Abian J, Stevenin J, Bach-Elias M. 2003. Roles of hnRNP A1, SR proteins, and p68 helicase in c-H-ras alternative splicing regulation. Mol Cell Biol 23: 2927-2941.

Han K, Yeo G, An P, Burge CB, Grabowski PJ. 2005. A combinatorial code for splicing silencing: UAGG and GGGG motifs. PLoS Biol 3: e158. doi: 10.1371/journal.pbio.0030158.

Honore B, Rasmussen HH, Vorum H, Dejgaard K, Liu X, Gromov P, Madsen P, Gesser B, Tommerup N, Celis JE. 1995. Heterogeneous nuclear ribonucleoproteins $\mathrm{H}, \mathrm{H}^{\prime}$, and $\mathrm{F}$ are members of a ubiquitously expressed subfamily of related but distinct proteins encoded by genes mapping to different chromosomes. J Biol Chem 270: $28780-28789$.

Hutchison S, LeBel C, Blanchette M, Chabot B. 2002. Distinct sets of adjacent heterogeneous nuclear ribonucleoprotein (hnRNP) A1/A2 binding sites control $5^{\prime}$ splice site selection in the hnRNP A1 mRNA precursor. J Biol Chem 277: 29745-29752. 
Jacquenet S, Mereau A, Bilodeau PS, Damier L, Stoltzfus CM, Branlant C. 2001. A second exon splicing silencer within human immunodeficiency virus type 1 tat exon 2 represses splicing of Tat mRNA and binds protein hnRNP H. J Biol Chem 276: 4046440475.

Jeronimo C, Forget D, Bouchard A, Li Q, Chua G, Poitras C, Therien C, Bergeron D, Bourassa S, Greenblatt J, et al. 2007. Systematic analysis of the protein interaction network for the human transcription machinery reveals the identity of the 7SK capping enzyme. Mol Cell 27: 262-274.

Kanopka A, Muhlemann O, Akusjarvi G. 1996. Inhibition by SR proteins of splicing of a regulated adenovirus pre-mRNA. Nature 381: 535-538.

Kashima T, Manley JL. 2003. A negative element in SMN2 exon 7 inhibits splicing in spinal muscular atrophy. Nat Genet 34: 460463.

Kashima T, Rao N, Manley JL. 2007. An intronic element contributes to splicing repression in spinal muscular atrophy. Proc Natl Acad Sci 104: 3426-3431.

Kohtz JD, Jamison SF, Will CL, Zuo P, Luhrmann R, GarciaBlanco MA, Manley JL. 1994. Protein-protein interactions and 5 -splice-site recognition in mammalian mRNA precursors. $\mathrm{Na}$ ture 368: 119-124.

Lavigueur A, La Branche H, Kornblihtt AR, Chabot B. 1993. A splicing enhancer in the human fibronectin alternate ED1 exon interacts with SR proteins and stimulates U2 snRNP binding. Genes \& Dev 7: 2405-2417.

Majewski J, Ott J. 2002. Distribution and characterization of regulatory elements in the human genome. Genome Res 12: 1827-1836.

Martinez-Contreras R, Fisette JF, Nasim FU, Madden R, Cordeau M, Chabot B. 2006. Intronic binding sites for hnRNP A/B and hnRNP F/H proteins stimulate pre-mRNA splicing. PLoS Biol 4: e21. doi: 10.1371/journal.pbio.0040021.

Martinez-Contreras R, Cloutier P, Shkreta L, Fisette JF, Revil T, Chabot B. 2007. hnRNP proteins and splicing control. Adv Exp Med Biol 623: 123-147.

Mauger DM, Lin C, Garcia-Blanco MA. 2008. hnRNP H and hnRNP F complex with Fox2 to silence fibroblast growth factor receptor 2 Exon IIIc. Mol Cell Biol 28: 5403-5419.

Mayeda A, Munroe SH, Caceres JF, Krainer AR. 1994. Function of conserved domains of hnRNP Al and other hnRNP A/B proteins. EMBO J 13: 5483-5495.

McCullough AJ, Berget SM. 1997. G triplets located throughout a class of small vertebrate introns enforce intron borders and regulate splice site selection. Mol Cell Biol 17: 4562-4571.

Min H, Chan RC, Black DL. 1995. The generally expressed hnRNP F is involved in a neural-specific pre-mRNA splicing event. Genes \& Dev 9: 2659-2671.

Nasim FU, Hutchison S, Cordeau M, Chabot B. 2002. High-affinity hnRNP A1 binding sites and duplex-forming inverted repeats have similar effects on $5^{\prime}$ splice site selection in support of a common looping out and repression mechanism. RNA 8: 1078-1089.

Nussinov R. 1987. (A)GGG(A), (A)CCC(A) and other potential 3' splice signals in primate nuclear pre-mRNA sequences. Biochim Biophys Acta 910: 261-270.

Nussinov R. 1988. Conserved quartets near $5^{\prime}$ intron junctions in primate nuclear pre-mRNA. J Theor Biol 133: 73-84.
Nussinov R. 1989. Conserved signals around the 5 ' splice sites in eukaryotic nuclear precursor mRNAs: G-runs are frequent in the introns and C in the exons near both 5' and 3' splice sites. J Biomol Struct Dyn 6: 985-1000.

Okunola HL, Krainer AR. 2009. Cooperative-binding and splicingrepressive properties of hnRNP A1. Mol Cell Biol 29: 5620-5631.

Pan Q, Shai O, Lee LJ, Frey BJ, Blencowe BJ. 2008. Deep surveying of alternative splicing complexity in the human transcriptome by high-throughput sequencing. Nat Genet 40: 1413-1415.

Paradis C, Cloutier P, Shkreta L, Toutant J, Klarskov K, Chabot B. 2007. hnRNP I/PTB can antagonize the splicing repressor activity of SRp30c. RNA 13: 1287-1300.

Pfleger KD, Seeber RM, Eidne KA. 2006. Bioluminescence resonance energy transfer (BRET) for the real-time detection of proteinprotein interactions. Nat Protoc 1: 337-345.

Romano M, Marcucci R, Buratti E, Ayala YM, Sebastio G, Baralle FE. 2002. Regulation of $3^{\prime}$ splice site selection in the 844ins68 polymorphism of the cystathionine $\beta$-synthase gene. J Biol Chem 277: 43821-43829.

Rooke N, Markovtsov V, Cagavi E, Black DL. 2003. Roles for SR proteins and hnRNP Al in the regulation of c-src exon N1. Mol Cell Biol 23: 1874-1884.

Simard MJ, Chabot B. 2002. SRp30c is a repressor of $3^{\prime}$ splice site utilization. Mol Cell Biol 22: 4001-4010.

Solovyev VV, Salamov AA, Lawrence CB. 1994. Predicting internal exons by oligonucleotide composition and discriminant analysis of spliceable open reading frames. Nucleic Acids Res 22: 5156-5163.

Ule J, Stefani G, Mele A, Ruggiu M, Wang X, Taneri B, Gaasterland T, Blencowe BJ, Darnell RB. 2006. An RNA map predicting Novadependent splicing regulation. Nature 444: 580-586.

Venables JP, Koh CS, Froehlich U, Lapointe E, Couture S, Inkel L, Bramard A, Paquet ER, Watier V, Durand M, et al. 2008. Multiple and specific mRNA processing targets for the major human hnRNP proteins. Mol Cell Biol 28: 6033-6043.

Wang ET, Sandberg R, Luo S, Khrebtukova I, Zhang L, Mayr C, Kingsmore SF, Schroth GP, Burge CB. 2008. Alternative isoform regulation in human tissue transcriptomes. Nature 456: 470-476.

Wang J, Dong Z, Bell LR. 1997. Sex-lethal interactions with protein and RNA. Roles of glycine-rich and RNA binding domains. J Biol Chem 272: 22227-22235.

Wang Z, Hoffmann HM, Grabowski PJ. 1995. Intrinsic U2AF binding is modulated by exon enhancer signals in parallel with changes in splicing activity. RNA 1: 21-35.

Xu Y, Piston DW, Johnson CH. 1999. A bioluminescence resonance energy transfer (BRET) system: Application to interacting circadian clock proteins. Proc Natl Acad Sci 96: 151-156.

Yeo G, Hoon S, Venkatesh B, Burge CB. 2004. Variation in sequence and organization of splicing regulatory elements in vertebrate genes. Proc Natl Acad Sci 101: 15700-15705.

Zahler AM, Damgaard CK, Kjems J, Caputi M. 2004. SC35 and heterogeneous nuclear ribonucleoprotein $\mathrm{A} / \mathrm{B}$ proteins bind to a juxtaposed exonic splicing enhancer/exonic splicing silencer element to regulate HIV-1 tat exon 2 splicing. J Biol Chem 279: 10077-10084.

Zuo P, Maniatis T. 1996. The splicing factor U2AF35 mediates critical protein-protein interactions in constitutive and enhancer-dependent splicing. Genes \& Dev 10: 1356-1368. 

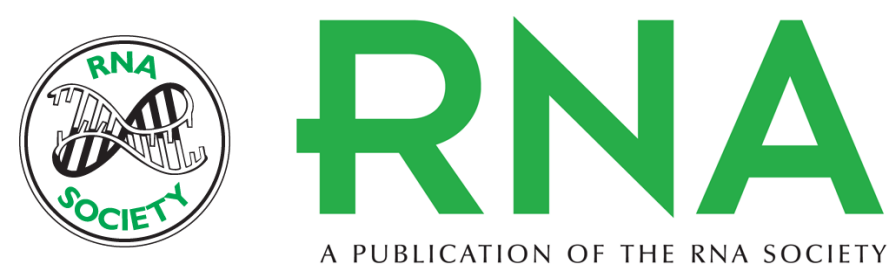

A PUBLICATION OF THE RNA SOCIETY

\section{hnRNP A1 and hnRNP H can collaborate to modulate 5' splice site selection}

Jean-François Fisette, Johanne Toutant, Samuel Dugré-Brisson, et al.

RNA 2010 16: 228-238 originally published online November 19, 2009

Access the most recent version at doi:10.1261/rna.1890310

\section{Supplemental http://rnajournal.cshlp.org/content/suppl/2009/11/06/rna.1890310.DC1 \\ Material}

References This article cites 58 articles, 33 of which can be accessed free at:

http://rnajournal.cshlp.org/content/16/1/228.full.html\#ref-list-1

\section{License}

Email Alerting Receive free email alerts when new articles cite this article - sign up in the box at the Service top right corner of the article or click here. 